\title{
Size of Muonium Hydride
}

\author{
M. SufFCZYŃSKI ${ }^{a}$, T. Kotowski ${ }^{b}$ AND L. WoLNIEWICZ ${ }^{c}$ \\ ${ }^{a}$ Institute of Physics, Polish Academy of Sciences \\ al. Lotników 32/46, 02-668 Warszawa, Poland \\ ${ }^{b}$ Institute of Experimental Physics, Warsaw University \\ Hoża 69, 00-681 Warszawa, Poland \\ ${ }^{c}$ Institute of Physics, Nicolas Copernicus University \\ Grudziądzka 5, 87-100 Toruń, Poland
}

(Received April 11, 2002; in final form June 18, 2002)

\begin{abstract}
Binding energy and expectation values of the interparticle distances of muonium hydride are calculated variationally with a wave function dependent exponentially on three interparticle distances.

PACS numbers: 36.10.Gv, 61.72.Vv
\end{abstract}

\section{Introduction}

The muonium hydride, $\mathrm{MuH}$, a system consisting of the muonium atom $\mathrm{Mu}$ and a hydrogen atom $\mathrm{H}$ is, like the positronium hydride and the $\mathrm{H}_{2}$ molecule, a four-particle neutral system. The proton to positron mass ratio is $m_{\mathrm{p}} / m_{\mathrm{e}}=$ 1836.1526675 , the proton to muon mass ratio is $m_{\mathrm{p}} / m_{\mu}=8.88024408$ [1].

In measurements of the muon spin precession in magnetic field [2-5], muonium is observed by means of its triplet state precession [2-4], and by the positive muon spin relaxation [3]. Muonium centers are investigated in insulators [3, 6, 7] and in elemental $[3,8]$ and compound $[3,7,9-14]$ semiconductors. Muonium formation in liquids [2], in gases [2, 3, 15] and in condensed rare gases [16], is of interest for a comparison of $\mathrm{Mu}$ formation rate constants with those of the hydrogen atom. Muonium hydride appears in the $\mathrm{Mu}$ reaction of hydrogen atom abstraction from linear molecules [2]. Muonium may become incorporated in a variety of molecules, and chemical consequences of replacement of hydrogen by muonium in a molecule can be observed $[2,3]$. Therefore, consideration of muonium bound to a neutral atom with an immobile nucleus is also of interest. 


\section{The wave function}

We calculated the ground state of muonium hydride with a variational wave function, symmetrized in the coordinates of the two electrons of the molecule, as described previously [17-21]. The wave function was a linear combination of monomials in four interparticle distances, with an overall exponential factor dependent on the three lepton distances from the most massive particle. The most massive particle was a proton in muonium hydride, $\mathrm{MuH}$, a deuteron with mass $m_{\mathrm{d}}=3670.4829550 m_{\mathrm{e}}$ in MuD [1], a triton with mass $m_{\mathrm{t}}=5496.918 m_{\mathrm{e}}$ in MuT $[22,23]$, and an immobile proton in $\mathrm{MuH}_{\infty}$. The corresponding reduced masses $\mu_{\mathrm{r}}$ of the muon are given in Table I. The variational wave function was [18]

$$
\begin{aligned}
& \Phi\left(r_{i j}\right)=\Psi_{\omega}\left(k r_{i j}\right), \\
& \Psi_{\omega}\left(r_{i j}\right)=\Psi_{\omega}\left(r_{1}, r_{2}, r_{12}, r_{3}, r_{13}, r_{23}\right)=\sum_{m n p q} c_{m n p q}\left(1+P_{12}\right)|m n p q\rangle, \\
& |m n p q\rangle=r_{1}^{m} r_{13}^{n} r_{3}^{p} r_{12}^{q} \exp \left(-\alpha r_{1}-\beta r_{2}-r_{3}\right)
\end{aligned}
$$

with nonnegative integers $m, n, p, q$, and the sum $m+n+p+q \leq \omega$. The $r_{1}$ and $r_{2}$ are the proton-electron distances, $r_{12}$ is the distance between the two electrons, $r_{13}$ and $r_{23}$ are the muon-electron distances, and $r_{3}$ is the proton-muon distance. $P_{12}$ permutes the indices 1,2 . The values of the nonlinear parameters $\alpha, \beta$ and of the scaling factor $k$ were determined by extrapolation from values of Refs. [18, 19, 21] and then by successive trials.

\section{The ground state energy}

The ground state energy of the muonium molecule was computed with a wave function comprising all monomials with the interparticle distance powers whose sum $\omega$ did not exceed 7 , which renders 330 linear terms.

\section{TABLE I}

The ground state energy of the muonium molecules.

\begin{tabular}{l|c|c|c}
\hline \hline & $\mu_{\mathrm{r}}$ & $-E$ & $D[\mathrm{eV}]$ \\
\hline $\mathrm{MuH}$ & 185.841 & 1.1389 & 3.8527 \\
$\mathrm{MuD}$ & 195.742 & 1.1395 & 3.8646 \\
$\mathrm{MuT}$ & 199.273 & 1.1397 & 3.8686 \\
$\mathrm{MuH}_{\infty}$ & 206.76827 & 1.1401 & 3.8766
\end{tabular}

Table I gives, for the muon reduced mass $\mu_{\mathrm{r}}$ (in units of electron mass), the computed ground state energy $E$ of the muonium molecule (in atomic units, 1 a.u. $=27.2113834 \mathrm{eV}[1]$ ), and the energy $D$, in $\mathrm{eV}$, of dissociation into a hydrogen atom and a muonium atom. For the triton mass we used the larger of 
the two quoted $[22,23]$ values, but this does not affect the value of the muon reduced mass within the digits retained by us.

\section{The interparticle distances}

Table II displays the expectation values of the interparticle distances in the muonium hydride computed with the optimized wave function, in units of the electron Bohr radius $a_{\mathrm{B}}=0.5291772083 \times 10^{-8} \mathrm{~cm} \mathrm{[1].}$

TABLE II

Expectation values of the interparticle distances.

\begin{tabular}{|c|c|c|c|c|}
\hline \multicolumn{5}{|c|}{ Inverses of the expectation values of the inverse distances } \\
\hline & $\left\langle r_{1}^{-1}\right\rangle^{-1}$ & $\left\langle r_{12}^{-1}\right\rangle^{-1}$ & $\left\langle r_{13}^{-1}\right\rangle^{-1}$ & $\left\langle r_{3}^{-1}\right\rangle^{-1}$ \\
\hline $\mathrm{MuH}$ & 1.115 & 1.781 & 1.157 & 1.466 \\
\hline $\mathrm{MuH}_{\infty}$ & 1.114 & 1.778 & 1.155 & 1.462 \\
\hline \multicolumn{5}{|c|}{ Expectation values of the first powers of distances } \\
\hline & $\left\langle r_{1}\right\rangle$ & $\left\langle r_{12}\right\rangle$ & $\left\langle r_{13}\right\rangle$ & $\left\langle r_{3}\right\rangle$ \\
\hline $\mathrm{MuH}$ & 1.600 & 2.283 & 1.670 & 1.534 \\
\hline $\mathrm{MuH}_{\infty}$ & 1.597 & 2.279 & 1.666 & 1.528 \\
\hline
\end{tabular}

Expectation values of the inverse interparticle distances, $\left\langle r^{-1}\right\rangle$, were computed in the calculation of the Coulomb potential energy of the system, with the 330-term wave function with optimized nonlinear parameters. Expectation values of the first positive power of distances, $\langle r\rangle$, were computed with $\omega=6$, i.e. with 210-term wave function.

The computed $\left\langle r_{12}^{-1}\right\rangle^{-1}$ in $\mathrm{MuH}$ is larger than in $\mathrm{H}_{2}$ by $3 \%$ [24]. The effective muon-electron separation estimated from a point-dipole model of anisotropy of the hyperfine coupling constant [25] for $\mathrm{Mu}$ in $\mathrm{Si}$ is between 1.8 and $2.1 \AA$, and larger in II-VI compound semiconductors [14], suggestive of an extended electron wave function.

At conclusion of the previous computation [26] was stressed the desirability of a computation with an improved precision. The present computations were performed with a REAL*16 precision. The results of the computations show that the muonium hydride is in its size similar to the neutral hydrogen molecule [18, 21, $24,26]$ rather than to a positronium hydride [20]. They quantitatively corroborate the opinion [3], supported by experimental evidence: "The importance of implanted-muon studies lies in the information that can be obtained via comparison of muon and proton behaviour. In aspects of chemical physics, the equivalent comparison is between muonium and hydrogen". 


\section{References}

[1] P.J. Mohr, B.N. Taylor, Rev. Mod. Phys. 72, 351 (2000).

[2] E. Roduner, Prog. React. Kinetics 14, 1 (1986).

[3] S.F.J. Cox, J. Phys. C, Solid State Phys. 20, 3187 (1987).

[4] B.D. Patterson, Rev. Mod. Phys. 60, 69 (1988).

[5] A. Weidinger, Ch. Niedermayer, A. Golnik, R. Simon, E. Recknagel, J.J. Budnick, B. Chamberland, C. Baines, Phys. Rev. Lett. 62, 102 (1989).

[6] J.H. Brewer, G.D. Morris, D.J. Arsenau, D.G. Eshchenko, V.G. Storchak, J. Bermejo, Physica B 289-290, 425 (2000).

[7] S.F.J. Cox, P.J.C. King, W.G. Williams, K.H. Chow, Th. Jestadt, W. Hayes, R.L. Lichti, C.R. Schwab, E.A. Davis, Physica B 289-290, 538 (2000).

[8] P.J.C. King, I. Yonenaga, Physica B 308-310, 546 (2001).

[9] S.F.J. Cox, E.A. Davis, S.P. Cottrell, P.J.C. King, J.S. Lord, J.M. Gil, H.V. Alberto, R.C. Vilao, J. Piroto Duarte, N. Ayres de Campos, A. Weidinger, R.L. Lichti, S.J.C. Irvine, Phys. Rev. Lett. 86, 2601 (2001).

[10] R.L. Lichti, S.F.J. Cox, M.R. Dawdy, T.L. Head, B. Hitti, R.J. Molnar, C. Schwab, R.P. Vaudo, Physica B 289-290, 542 (2000).

[11] M.R. Dawdy, R.L. Lichti, S.F.J. Cox, T.L. Head, C. Schwab, Physica B 289-290, $546(2000)$.

[12] E.S. Bates, R.L. Lichti, S.F.J. Cox, C. Schwab, Physica B 289-290, 550 (2000).

[13] J.M. Gil, H.V. Alberto, R.C. Vilao, J. Piroto Duarte, P.J. Mendes, N. Ayres de Campos, A. Weidinger, J. Krauser, Ch. Niedermayer, S.F.J. Cox, Physica B 289-290, 563 (2000).

[14] J.S. Lord, S.P. Cottrell, P.J.C. King, H.V. Alberto, N. Ayres de Campos, J.M. Gil, J. Piroto Duarte, R.C. Vilao, R.L. Lichti, S.K.L. Sjue, B.A. Bailey, A. Weidinger, E.A. Davis, S.F.J. Cox, Physica B 308-310, 920 (2001).

[15] D. J. Arsenau, M. Senba, J.J. Pan, D.G. Fleming, Physica B 289-290, 503 (2000).

[16] D.G. Eshchenko, V.G. Storchak, J.H. Brewer, G.D. Morris, M.A. Clarker-Gayther, S.P. Cottrell, S.F.J. Cox, J.S. Lord, V.N. Gorelkin, Physica B 289-290, 418 (2000)

[17] B.A. Page, P.A. Fraser, J. Phys. B, At. Mol. Phys. 7, L389 (1974).

[18] B. Stébé, G. Munschy, Solid State Commun. 35, 557 (1980).

[19] F. Dujardin, B. Stébé, Phys. Status Solidi B 140, K117 (1987).

[20] Y.K. Ho, Phys. Rev. A 34, 609 (1986).

[21] M. Suffczyński, L. Wolniewicz, Phys. Rev. B 40, 6250 (1989).

[22] K. Szalewicz, H.J. Monkhorst, W. Kolos, A. Scrinzi, Phys. Rev. A 36, 5494 (1987).

[23] K. Szalewicz, B. Jeziorski, A. Scrinzi, X. Zhao, R. Moszyński, W. Kolos, P. Froelich, H.J. Monkhorst, A. Velenik, Phys. Rev. A 42, 3768 (1990).

[24] L. Wolniewicz, J. Chem. Phys. 45, 515 (1966).

[25] S.F.J. Cox, M.C.R. Symons, Chem. Phys. Lett. 126, 516 (1986).

[26] M. Suffczyński, L. Wolniewicz, Acta Phys. Pol. A 83, 157 (1993). 